\title{
On the Multicast Capacity of Wireless Ad Hoc Networks with Network Coding
}

\author{
Zheng Wang, Shirish S. Karande, Hamid R. Sadjadpour, and J. J. Garcia-Luna-Aceves
}

\begin{abstract}
In this paper, we study the contribution of network coding (NC) in improving the multicast capacity of random wireless ad hoc networks when nodes are endowed with multi-packet transmission (MPT) and multi-packet reception (MPR) capabilities. We show that a per session throughput capacity of $\Theta\left(n T^{3}(n)\right)$ can be achieved as a tight bound when each session contains a constant number of sinks where $n$ is the total number of nodes and $T(n)$ is the transmission range ${ }^{1}$. Surprisingly, an identical order capacity can be achieved when nodes have only MPR and MPT capabilities. This result proves that NC does not contribute to the order capacity of multicast traffic in wireless ad hoc networks when MPR and MPT are used in the network. The result is in sharp contrast to the general belief (conjecture) that $\mathrm{NC}$ improves the order capacity of multicast. Furthermore, if the communication range is selected to guarantee the connectivity in the network, i.e., $\Omega(\sqrt{\log n / n})=$ $T(n)=O(\log \log n / \log n)$, then the combination of MPR and MPT achieves a throughput capacity of $\Theta\left(\log ^{\frac{3}{2}} n / \sqrt{n}\right)$ which provides an order capacity gain of $\Theta\left(\log ^{2} n\right)$ compared to the pointto-point multicast capacity with the same number of destinations.
\end{abstract}

Index Terms: Capacity, multicast, network coding, wireless ad hoc networks.

\section{INTRODUCTION}

The seminal work by Gupta and Kumar [1] has sparked a significant interest in investigating the fundamental capacity limits of wireless ad hoc networks. Several techniques [2]-[4] have been developed with the objective of improving the capacity of wireless ad hoc networks. Network coding (NC), which was originally proposed by Ahlswede et al. in [5], is one such technique. Unlike traditional store-and-forward routing, network coding scheme encodes the messages received at intermediate nodes, prior to forwarding them to subsequent nexthop neighbors. Ahlswede et al. [5] showed that network coding can achieve a multicast flow equal to the min-cut for a single source and under the assumptions of a directed graph. This and other works in network coding (NC) [6]-[8] have motivated a large number of researchers to investigate the impact of $\mathrm{NC}$ in increasing the throughput capacity of wireless ad hoc net-

Manuscript received April 20 2010; approved for publication by Song Chong, Division III Editor, December 11, 2010.

Z. Wang and H. R. Sadjadpour are with the Department of Electrical Engineering, University of California, Santa Cruz, 1156 High Street, Santa Cruz, CA 95064, USA, email: \{wzgold, hamid\}@soe.ucsc.edu.

S. S. Karande is with the Philips Research Bangalore, No.1, Murphy Road, Ulsoor, Bangalore, 560 008, India, email: shirish.karande@philips.com.

J. J. Garcia-Luna-Aceves is with the Department of Computer Engineering, University of California, Santa Cruz, 1156 High Street, Santa Cruz, CA 95064, USA and the Palo Alto Research Center (PARC), 3333 Coyote Hill Road, Palo Alto, CA 94304, USA, email: jj@soe.ucsc.edu.

${ }^{1} T(n)$ is constrained by the decoding/encoding complexity of the nodes in the network. works. However, Liu et al. [9] recently showed that NC does not increase the order of the throughput capacity for multi-pair unicast traffic. Nevertheless, a number of efforts (analog network coding [10], physical network coding [11]) have continued the quest for improving the multicast capacity of ad-hoc networks by using NC. Despite the claims of throughput improvement by such studies, the impact of $\mathrm{NC}$ on the multicast scaling law has remained uncharacterized.

Promising approaches [10], [11] implicitly assume the combination of NC (transmitting multiple packets encoded in a single transmission) with multi-packet transmission (MPT) and multi-packet reception (MPR) [12]-[14] (i.e., the ability to successfully transceive multiple concurrent transmissions by employing physical-layer interference cancelation techniques). We consider MPT and MPR as an equivalent model (in terms of transmitter or receiver point of view respectively) for fully connected graph when transceiver range $T(n)=1$ or partially connected graph when $T(n)<1$. Note that the selection of $T(n)$ in MPT and MPR depends on the decoding/encoding complexity of the nodes in the network. One effective technique to implement MPT and MPR is to utilize beamforming. The detail implementation of this technique has been discussed extensively in [15] and [16]. MPR has been shown to increase the capacity regions of ad hoc networks [17], and very recently GarciaLuna-Aceves et al. [18] have shown that the order capacity in wireless ad hoc networks subject to multi-pair unicast traffic is increased with MPR. These prior efforts raise three important questions: (a) What is the order of multicast throughput achieved by the combination of NC with MPT and MPR? (b) Does this combination provide us with an order gain (instead of a constant) over traditional techniques based on routing and point-topoint communication? (c) If yes, what exactly leads to this gain? Is NC necessary or does the combination of MPT and MPR suffice? It is important to note that combination of NC with MPT and MPR has been considered in literature [12], [13], and [14] without explicitly using these terms and it is therefore important to investigate the above questions.

In this work, we address the above three questions. The answers can be summarized by our main results.

- When each multicast group consists of a constant number of sinks, the combination of NC, MPT and MPR provides a per session throughput capacity of $\Theta\left(n T^{3}(n)\right)$, where $T(n)$ is the communication range.

- This scaling law represents an order gain of $\Theta\left(n^{2} T^{4}(n)\right)$ over a combination of routing and point-to-point communication.

- The combination of only MPT and MPR is sufficient to achieve a per-session multicast throughput order of $\Theta\left(n T^{3}(n)\right)$. Consequently, $\mathrm{NC}$ does not contribute to the multicast capacity when MPR and MPT are used in the network! 
The remainder of this paper is organized as follows. In Section II, we give an overview of capacity analysis for NC, MPT, MPR, and other existing techniques. In Section III, we introduce the models we used. In Sections IV and V, we give our main results with MPT and MPR when network coding is not used and used, respectively. We conclude our paper in Section VI.

\section{LITERATURE REVIEWS}

Gupta and Kumar in their seminal paper [1] proved that the throughput capacity in wireless ad hoc network is not scalable. Subsequently, many researchers have focused on identifying techniques that could alter this conclusion. Recently, Ozgur et al. [4] proposed a hierarchical cooperation technique based on virtual MIMO to achieve linear per source-destination capacity. Cooperation can be extended to the simultaneous transmission and reception at the various nodes in the network, which is called many-to-many communication and can result in significant improvement in capacity [3].

There has been some work focused on the multicast capacity in wireless ad hoc networks. Since the original paper by Ahlswede et al. [5], most of the research on network coding has focused on directed networks, where each communication link has a fixed direction. $\mathrm{Li}$ and $\mathrm{Li}$ [19] were the first to study the benefits of network coding in undirected networks, where each communication link is bidirectional. Their result [19] shows that, for a single unicast or broadcast session, there is no improvement with respect to throughput due to network coding. In the case of a single multicast session, such an improvement is bounded by a factor of two. Meanwhile, the authors of [12], [13], and [14] studied the throughput capacity of NC in wireless ad hoc networks. However, the authors of [12], [13], and [14] employ network models that are fundamentally inconsistent with the more commonly accepted assumptions of ad-hoc networks [1]. Specifically, the model constraints of [12]-[14], [19], and [20] differ as follows: All the prior works assume a single source for unicast, multicast, or even broadcast. Aly et al. [13] and Kong et al. [14] differentiate the total nodes into source set, relay set and destination set. They do not allow all of the nodes to concurrently serve as sources, relays or destinations, as allowed in the work by Gupta and Kumar [1]. Furthermore, these results do not consider the impact of interference in wireless ad hoc networks.

Specifically, If sources, relays and destinations are strictly different, the authors of [13] use max-flow min-cut of network coding. But if they are not, it will make the problem much harder to solve. This paper discussed the impact of MPT and/or MPR ability while [13] is totally connected graph with no transmission range flavor in wireless network environment.

In the absence of interference, the communication scenario equates an ideal case where a node can simultaneously transmit and receive from multiple nodes. Interference cancelation techniques such as MPT and MPR indeed enable nodes with the ability of multi-point communication within a communication range of $T(n)$. Thus, the model assumptions in [12], [13], and [14] at the very least assume that nodes are capable of MPT and MPR. Similarly, works such as physical-layer network coding
(PNC) [11] by Zhang et al. and analog network coding [10] by Katti et al. also implicitly assume the ability of MPT and MPR.

Finally, beyond the work of [21], we revised considerately upper bound proof as area argument instead of tree one and used bins and balls argument for lower bound compared with [21]. The authors of [22] and [23] analyzed the contribution of NC itself while this paper proves that utilization of MPT and/or MPR with NC in wireless networks achieves the same gain as MPT and/or MPR.

\section{NETWORK MODEL, DEFINITIONS, AND PRELIMINARIES}

We assume a random wireless ad hoc network with $n$ nodes distributed uniformly in a unit-square network area. Our capacity analysis is based on the protocol model for dense and static networks, introduced by Gupta and Kumar [1]. The case of what we call point-to-point communication corresponds to the original protocol model.

Definition 1: The protocol model of point-to-point communication.

All nodes use a common transmission range $r(n)$ for all their communication. Node $X_{i}$ can successfully transmit to node $X_{j}$ if for any node $X_{k}, k \neq i$, that transmits at the same time as $X_{i}$ it is true that $\left|X_{i}-X_{j}\right| \leq r(n)$ and $\left|X_{k}-X_{j}\right| \geq(1+\Delta) r(n)$, where, $\Delta$ is a constant guard distance.

We make the following extensions to account for MPT and MPR capabilities at the transmitters and receivers, respectively. In wireless ad hoc networks with MPT (MPR) capability, any transmitter (receiver) node can transmit (receive) different information simultaneously to (from) multiple nodes within the circle whose radius is $T(n)$ [18]. For the rest of this paper, we assume for simplicity that $T(n)=r(n)$. We assume that it is possible to achieve ideal MPT and MPR. In a non-ideal scenario, realization of MPT or MPR leads to a loss in the rate provided to an individual receiver. Certain special cases in the rate region can also be expressed as reduction in transmission range, i.e., $T(n)<r(n)$. In idealized conditions, $r(n)=T(n)$. We further assume that nodes cannot transmit and receive at the same time, which is equivalent to half-duplex communications [1]. From system point of view, MPT and MPR are dual if we consider the source and destination duality.

Definition 2: Feasible throughput capacity.

In a wireless ad hoc network with $n$ nodes where each source transmits its packets to $m$ destinations, a throughput of $C_{m}(n)$ bits per second for each node is feasible if there is a spatial and temporal scheme for scheduling transmissions, such that, by operating the network in a multi-hop fashion and buffering at intermediate nodes when awaiting transmission, every node can send $C_{m}(n)$ bits per second on average to its $m$ chosen destination nodes. That is, there is a $T_{0}<\infty$ such that in every time interval $\left[(i-1) T_{0}, i T_{0}\right]$ every node can send $T_{0} C_{m}(n)$ bits to its corresponding destination nodes.

Definition 3: Order of throughput capacity. $C_{m}(n)$ is said to be of order $\Theta(f(n))$ bits/second if there exist deterministic positive constants $c$ and $c^{\prime}$ such that

$$
\left\{\begin{array}{r}
\lim _{n \rightarrow \infty} \operatorname{Prob}\left(C_{m}(n)=c f(n) \text { is feasible }\right)=1 \\
\liminf \operatorname{in}_{n \rightarrow \infty} \operatorname{Prob}\left(C_{m}(n)=c^{\prime} f(n) \text { is feasible }\right)<1
\end{array}\right.
$$




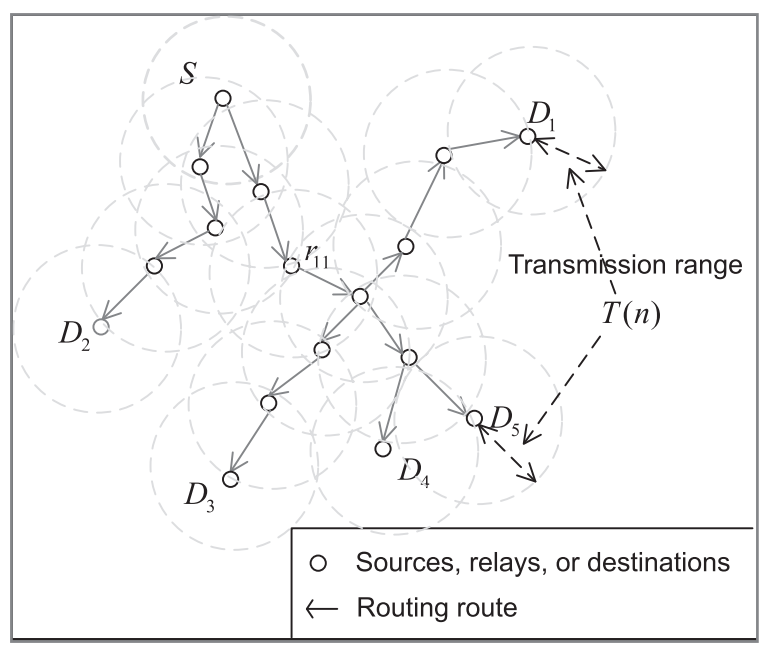

Fig. 1. Area coverage by one multicast tree.

Definition 4: Euclidean minimum spanning tree (EMST). Consider a connected undirected graph $G=(V, E)$, where $V$ and $E$ are sets of vertices and edges in the graph $G$, respectively. The EMST of $G$ is a spanning tree of $G$ with the minimum sum of Euclidean distances between connected vertices of this tree.

Definition 5: Minimum Euclidean multicast tree (MEMT). The network can be described a connected graph $G_{T(n)}\left(V, E_{T(n)}\right)$ where the vertices represent the nodes in the network and the edge set is determined by the transmission range $T(n)$ such that there is an edge between any two vertices separated by a distance less than $T(n)$. Let, $s \in V$ be a source and $D_{s}=d_{s_{1}}, d_{s_{2}}, \cdots, d_{s_{m}}$ be $m$ multicast destinations, then an $\operatorname{MEMT}(T(n))$ is an edge-minimal tree in $G_{T(n)}$ that spans $D_{s} \bigcup s$.

Definition 6: Minimum area multicast tree (MAMT).

We define the area of any tree in $G_{T(n)}$ as the area of the region formed by taking the union of circles, of radius $T(n)$, placed at each node in the tree. Let, $s \in V$ be a source and $D_{s}=d_{s_{1}}, d_{s_{2}}, \cdots, d_{s_{m}}$ be $m$ multicast destinations, then an $\operatorname{MAMT}(T(n))$ is an area-minimal tree in $G_{T(n)}$ that spans $D_{s} \cup s$.

Note that EMST and MEMT are spanning trees which includes only source and destinations, while MAMT is related to a real routing tree which includes relays.

Definition 7: Total active area $(T A A(\Delta, T(n)))$.

The $\operatorname{TAA}(\Delta, T(n))$ is the total area of the network multiplied by the average maximum number of simultaneous transmissions and receptions inside a communication region of $\Theta\left(T^{2}(n)\right)$.

It can be shown that this value has an upper bound of $O(1)$, $O\left(n T^{2}(n)\right)$ and $O\left(n^{2} T^{4}(n)\right)$ for point-to-point, MPR (or MPT) and MPR combined with MPT respectively.

In the rest of this paper, $\left\|T_{r}\right\|$ denotes the total Euclidean distance of a tree $T_{r} ; \# T_{r}$ is used to denote the total number of vertices (nodes) in a tree $T_{r} ; S\left(T_{r}\right)$ denotes the area of tree $T_{r}$ covered; and $\overline{\left\|T_{r}\right\|}$ is used for the statistical average of the total Euclidean distance of a tree.

To compute the multicast capacity, we use the relationship between MAMT and EMST. Steele [24] determined a tight bound for $\overline{\| \text { EMST } \|}$ for a group of $m$ nodes when $m \gg 1$, which we restate in the following lemma.
Lemma 1: Let $f(x)$ denote the node probability distribution function in the network area. Then, for large values of $m$ and $d>1$, the $\overline{\| \text { EMST } \|}$ is tight bounded as

$$
\overline{\| \text { EMST } \|}=\Theta\left(c(d) m^{\frac{d-1}{d}} \int_{R^{d}} f(x)^{\frac{d-1}{d}} d x\right)
$$

where $d$ is the dimension of the network. Note that both $c(d)$ and the integral are constant values and not functions of $m$. When $d=2$, then $\overline{\| \text { EMST } \|}=\Theta(\sqrt{m})$.

Given that the distribution of nodes in a random network is uniform, if there are $n$ nodes in a unit square, then the density of nodes equals $n$. Hence, if $|S|$ denotes the area of space region $S$, the expected number of the nodes, $E\left(N_{S}\right)$, in this area is given by $E\left(N_{S}\right)=n|S|$. Let $N_{j}$ be a random variable defining the number of nodes in $S_{j}$. Then, for the family of variables $N_{j}$, we have the following standard results known as the Chernoff bounds [25]:

Lemma 2: Chernoff bound

- For any $\delta>0, P\left[N_{j}>(1+\delta) n\left|S_{j}\right|\right]<\left(\frac{e^{\delta}}{(1+\delta)^{1+\delta}}\right)^{n\left|S_{j}\right|}$.

- For any $0<\delta<1, P\left[N_{j}<(1-\delta) n\left|S_{j}\right|\right]<e^{-\frac{1}{2} n\left|S_{j}\right| \delta^{2}}$.

Combining these two inequalities we have, for any $0<\delta<1$ :

$$
P\left[\left|N_{j}-n\right| S_{j}||>\delta n\left|S_{j}\right|\right]<e^{-\theta n\left|S_{j}\right|}
$$

where $\theta=(1+\delta) \ln (1+\delta)-\delta$ in the case of the first bound, and $\theta=\delta^{2} / 2$ in the case of the second bound.

Therefore, for any $\theta>0$, there exist constants such that deviations from the mean by more than these constants occur with probability approaching zero as $n \rightarrow \infty$. It follows that, with high probability (w.h.p.) ${ }^{2}$, we can get a very sharp concentration on the number of nodes in an area, so we can find the achievable lower bound w.h.p., provided that the upper bound (mean) is given. In the following sections, we first derive the upper bound, and then use the Chernoff bound to prove the achievable lower bound.

In [5], it was proved that the max-flow min-cut is equal to multicast capacity of a directed graph with single source. The directed graph model is more applicable for wired networks. However, in this work we wish to study the utility of NC in a wireless environment where links are bidirectional [12], [13].

In a single-source network, the cut capacity is equal to the maximum flow. Thus, [13] provides an upper bound on the multicast capacity of a network with single source and NC+MPT+MPR capability. However, in [12], [13], and [14], the source, relays and destinations are strictly different and information can not be transmitted directly towards the destinations. These two assumptions will be eventually relaxed in this paper.

Before we analyze the scaling law of throughput capacity with NC, MPT and MPR in wireless ad hoc networks, we want to illustrate one example to claim the consequences of MPT and MPR as Fig. 2. From this figure, we observe that combination of MPT, MPR and NC can increase the information flow and the gain is equivalent to only combining MPT and MPR. Intuitively, we can conjecture that the gain of NC is given actually by MPT

\footnotetext{
${ }^{2}$ An event happens with high probability if the probability of this event is greater than $1-1 / n$ when $n$ goes to infinity.
} 


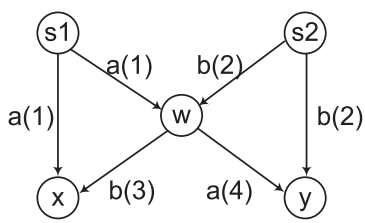

(a)

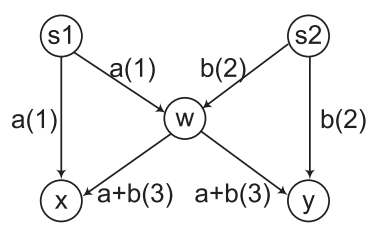

(b)

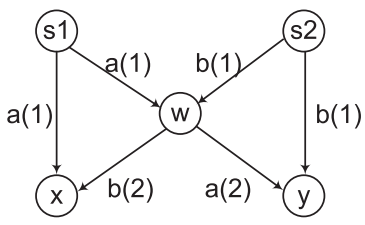

(c)

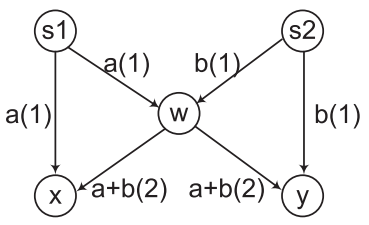

(d)

Fig. 2. One example for point-to-point communication, MPT, MPR, and NC: (a) Point-to-point (4), (b) NC (3), (c) MPT + MPR (2), and (d) MPT + $\mathrm{MPR}+\mathrm{NC}(2)$

Table 1. Abbreviation table.

\begin{tabular}{|c|c|}
\hline EMST & Euclidean minimum spanning tree \\
\hline MEMT & Minimum Euclidean multicast tree \\
\hline MEMTC & Minimum Euclidean multicast tree cells \\
\hline MAMT & Minimum area multicast tree \\
\hline TAA & Total active area \\
\hline$r(n)$ & $\begin{array}{r}\text { Transmission range in } \\
\text { point-to-point communication }\end{array}$ \\
\hline$T(n)$ & Transceiver range in MPT and MPR \\
\hline
\end{tabular}

and/or MPR which will be proved in the following sections. In Section IV and V, we will prove that the scaling law of MPT and MPR abilities without and with NC respectively, and then show they are the same order.

Table 1 summarizes all the abbreviations that are used in this paper.

\section{THE THROUGHPUT CAPACITY WITH MPT AND MPR}

In this section, we compute the scaling laws in random geometric graphs when nodes are endowed with MPR and MPT capabilities. Our approach is based on the results in [26] for pointto-point communication and extending it to MPR and MPT cases.

\section{A. Upper Bound}

The following Lemma provides an upper bound for the per-session capacity as a function of $\overline{\operatorname{TAA}(\Delta, T(n))}$ and $\overline{S(\text { MAMT }(T(n)))}$. Essentially, $\overline{S(\operatorname{MAMT}(T(n)))}$ equals the minimum area consumed to multicast a packet to $m$ destinations (see Fig. 1), and $\overline{\operatorname{TAA}(\Delta, T(n))}$ represents the maximum area which can be supported when MPT and MPR are used.

Lemma 3: In random dense wireless ad hoc networks, the per-node throughput capacity of multicast with MPT and MPR is given by $O\left(\frac{1}{n} \frac{\overline{\operatorname{TAA}(\Delta, T(n))}}{\overline{S(\operatorname{MAMT}(T(n)))}}\right)$.

Proof: With MPT and MPR, we observe that $\overline{S(\text { MAMT }(T(n)))}$ represents the total area required to transmit information from a multicast source to all its $m$ destinations. The ratio between average total active area, $\overline{\operatorname{TAA}(\Delta, T(n))}$, and $\overline{S(\text { MAMT }(T(n)))}$ represents the average number of simultaneous multicast communications that can occur in the network. Normalizing this ratio by $n$ provides per-node capacity.

Lemma 3 provides the upper bound for the multicast throughput capacity with MPT and MPR as a function of $\overline{S(\operatorname{MAMT}(T(n)))}$ and $\overline{\operatorname{TAA}(\Delta, T(n))}$. In order to compute the upper bound, we derive the upper bound of $\overline{\operatorname{TAA}(\Delta, T(n))}$ and the lower bound of $\overline{S(\text { MAMT }(T(n)))}$. Combining these results provides an upper bound for the multicast throughput capacity with MPT and MPR.

Lemma 4: The average area of a multicast tree with transmission range $T(n), \overline{S(\text { MAMT }(T(n)))}$ is lower bounded by $\Omega(T(n))$, when $m$ is a constant value.

Proof: From [27], it can be deduced that $\overline{S(\operatorname{MAMT}(T(n)))}$ is lower bounded as $\Omega(\overline{\|\mathrm{EMST}\|} T(n))$. Even for the case of the minimum value for $T(n)$ to assure connectivity, this upper bound is guaranteed for constant values of $m$. Lemma 1 states that $\overline{\| \text { EMST } \|}=\Theta(\sqrt{m})=\Theta(1)^{*}$. The proof follows immediately.

Lemma 5: The average total active area, $\overline{\operatorname{TAA}(\Delta, T(n))}$, has the following upper bound in networks with MPT and MPR.

$$
\overline{\operatorname{TAA}(\Delta, T(n))}=O\left(n^{2} T^{4}(n)\right) .
$$

Proof: As discussed earlier, the $\overline{\mathrm{TAA}(\Delta, T(n))}$ for pointto-point communication is equal to 1 since for each circle of radius $T(n)$, there is only a single pair of transmitter-receiver nodes (see Fig. 3(a)). For the case of MPR and MPT, the number of nodes in a circle of radius $T(n)$ is upper bounded as $O\left(n T^{2}(n)\right)$. This is also upper bound for the number of transmitters or receivers in this region. The upper bound for $\overline{\operatorname{TAA}(\Delta, T(n))}$ is achieved when the maximum number of transmitters and receivers are employed in this circle. Fig. 3(b) demonstrates an example that can achieve this upper bound simultaneously for transmitters and receivers. Let a circle of radius $T(n) / 2$ located at the center of another circle of radius $T(n)$. Note that with this construction, any two nodes inside the small circle are connected. If we randomly assign half of the nodes inside the small circle as transmitters and the other half as receiver nodes, then the average number of transmitters and receivers in this circle are proportional to $\Theta\left(n T^{2}(n)\right)$. Given the fact that this value also is the maximum possible number of transmitter and receiver nodes, the result follows immediately.

Combining Lemmas 3, 4, and 5, we can compute the upper bound for multicast capacity of MPT and MPR.

Theorem 1: In wireless ad hoc networks with MPT and MPR, the upper bound on the per-node throughput capacity of multicast with constant number of destinations is

$$
C_{m}(n)=O\left(n T^{3}(n)\right)
$$

${ }^{*} m$ is a constant value. 


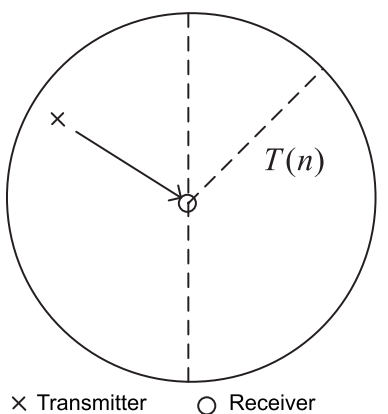

(a)

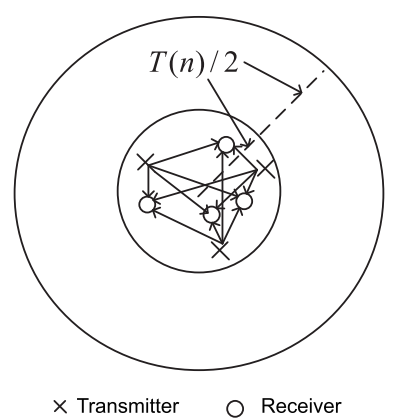

(b)
Fig. 3. Upper bound of total available area based on protocol model: (a) Point-to-point communication and (b) MPT and MPR.

\section{B. Lower Bound}

To derive an achievable lower bound, we use a TDMA scheme for random dense wireless ad hoc networks similar to the approach used in [28] and [29].

We first divide the network area into square cells. Each square cell has an area of $T^{2}(n) / 2$, which makes the diagonal length of square equal to $T(n)$, as shown in Fig. 4. Under this condition, connectivity inside all cells is guaranteed and all nodes inside a cell are within communication range of each other. We build a cell graph over the cells that are occupied with at least one vertex (node). Two cells are connected if there exist a pair of nodes, one in each cell, that are less than or equal to $T(n)$ distance apart. Because the whole network is connected when $T(n)=r(n)=$ $\Omega(\sqrt{\log n / n})$, it follows that the cell graph is connected [28], [29].

To satisfy the MPT and MPR protocol model, we organize cells in groups so that simultaneous transmissions within each group does not violate the conditions for successful communication in the MPT and MPR protocol model. Let $L$ represent the minimum number of cell separations in each group of cells that communicate simultaneously. Utilizing the protocol model, $L$ satisfies the following condition:

$$
L=\left\lceil 1+\frac{T(n)+(1+\Delta) T(n)}{T(n) / \sqrt{2}}\right\rceil=\lceil 1+\sqrt{2}(2+\Delta)\rceil .
$$

If we divide time into $L^{2}$ time slots and assign each time slot to a single group of cells, interference is avoided and the protocol model is satisfied. The separation example can be shown for the upper two receiver circles in Fig. 4. For the MPT and MPR protocol model, the distance between two adjacent receiving nodes is $(2+\Delta) T(n)$. Because this distance is smaller than $(L-1) T(n)$, this organization of cells guarantees that the MPT and MPR protocol model is satisfied. Fig. 4 represents one of these groups with a cross sign inside those cells for $L=4$. We can derive an achievable multicast capacity for MPT and MPR by taking advantage of this cell arrangement and TDMA scheme. The capacity reduction caused by the TDMA scheme is a constant factor and does not change the order capacity of the network.

Next, our objective is to find an achievable lower bound using

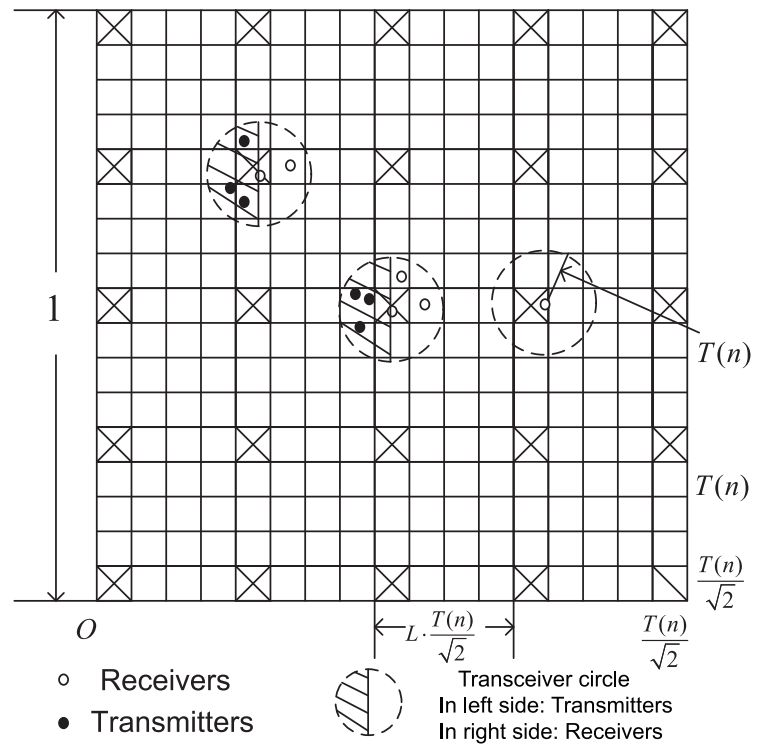

Fig. 4. Cell construction used to derive a lower bound on capacity.

the Chernoff bound, such that the distribution of the number of edges in this unit space is sharply concentrated around its mean, and hence the actual number of simultaneous transmissions occurring in the unit space in a randomly chosen network is indeed $\Theta\left(n^{2} T^{2}(n)\right)$ w.h.p..

Lemma 6: The circular area of radius $T(n)$ corresponding to the transceiver range of any node $j$ in the cross area in Fig. 4 contains $\Theta\left(n T^{2}(n)\right)$ nodes w.h.p., and is uniformly distributed for all values of $j, 1 \leq j \leq 1 /(L T(n) / \sqrt{2})^{2}$.

Proof: The statement of this lemma can be expressed as

$$
\lim _{n \rightarrow \infty} P\left[\bigcap_{j=1}^{\frac{1}{(L T(n) / \sqrt{2})^{2}}}\left|N_{j}-E\left(N_{j}\right)\right|<\delta E\left(N_{j}\right)\right]=1
$$

where $N_{j}$ and $E\left(N_{j}\right)$ are the random variables that represent the number of transmitters in the receiver circle of radius $T(n)$ centered by the receiver $j$ and the expected value of this random variable, respectively and $\delta$ is a positive arbitrarily small value close to zero.

From the Chernoff bound in (3), for any given $0<\delta<1$, we can find $\theta>0$ such that $P\left[\left|N_{j}-E\left(N_{j}\right)\right|>\delta E\left(N_{j}\right)\right]<$ $e^{-\theta E\left(N_{j}\right)}$. Thus, we can conclude that the probability that the value of the random variable $N_{j}$ deviates by an arbitrarily small constant value from the mean tends to zero as $n \rightarrow \infty$. This is a key step in showing that when all the events $\bigcap_{j=1}^{\overline{(L T(n) / \sqrt{2})^{2}}} \mid N_{j}-$ $E\left(N_{j}\right) \mid<\delta E\left(N_{j}\right)$ occur simultaneously, then all $N_{j}$ 's converge uniformly to their expected values. Utilizing the union bound, we arrive at

$$
\begin{aligned}
& P\left[\bigcap_{j=1}^{\frac{1}{(L T(n) / \sqrt{2})^{2}}}\left|N_{j}-E\left(N_{j}\right)\right|<\delta E\left(N_{j}\right)\right] \\
& =1-P\left[\bigcup_{j=1}^{\frac{1}{(L T(n) / \sqrt{2})^{2}}}\left|N_{j}-E\left(N_{j}\right)\right|>\delta E\left(N_{j}\right)\right]
\end{aligned}
$$




$$
\begin{aligned}
& \geq 1-\sum_{j=1}^{\frac{1}{(L T(n) / \sqrt{2})^{2}}} P\left[\left|N_{j}-E\left(N_{j}\right)\right|>\delta E\left(N_{j}\right)\right] \\
& >1-\frac{1}{(L T(n) / \sqrt{2})^{2}} e^{-\theta E\left(N_{j}\right)} .
\end{aligned}
$$

Given that $E\left(N_{j}\right)=\pi n T^{2}(n)$, then we have

$$
\begin{aligned}
& \lim _{n \rightarrow \infty} P\left[\bigcap_{j=1}^{\frac{1}{(L T(n) / \sqrt{2})^{2}}}\left|N_{j}-E\left(N_{j}\right)\right|<\delta E\left(N_{j}\right)\right] \\
& \geq 1-\lim _{n \rightarrow \infty} \frac{1}{(L T(n) / \sqrt{2})^{2}} e^{-\theta \pi n T^{2}(n)} .
\end{aligned}
$$

Utilizing the connectivity criterion, $\lim _{n \rightarrow \infty} \frac{e^{-\theta \pi n T^{2}(n)}}{T^{2}(n)} \rightarrow 0$, which finishes the proof.

Furthermore, we can arrange all of the nodes in the left side of the corresponding transceiver circle be the transmitters, and all of the nodes in the right side of the corresponding transceiver circle be the receivers. Thus, we arrive at the following lemma.

Lemma 7: In the unit square area for a wireless ad hoc network shown in Fig. 4, the total number of transmitter-receiver links (simultaneous transmissions) is $\Omega\left(n^{2} T^{2}(n)\right)$.

Proof: From Lemma 6, for any node in the cross cell in the whole network shown in Fig. 4, there are $\Theta\left(n T^{2}(n)\right)$ nodes in the transceiver circle. We divided the total nodes into two categories, transmitters in the left of the transceiver circles and receivers in the right of the transceiver circles. To guarantee all of the transmitters and receivers are in the transceiver range, we only consider the nodes in the circle with radius $T(n) / 2$. Because of the MPT and MPR capabilities, so that every transmitter in the left of the transceiver circle with $T(n) / 2$ radius can transmit successfully to every receiver in the right, then the total number of successful transmissions is $\pi^{2} n^{2} T^{4}(n) / 16$ which is the achievable lower bound. The actual number of the transmissions can be much larger than this because we only consider $T(n) / 2$ instead of $T(n)$. Using the Chernoff bound in (3) and Lemma 6, we can show that the total number of successful transmissions is

$$
\Omega\left(\frac{\pi^{2} n^{2} T^{4}(n)}{16(L T(n) / \sqrt{2})^{2}}\right)=\Omega\left(n^{2} T^{2}(n)\right) .
$$

The above results enables us to obtain the following achievable lower bound.

Let us define \#MEMTC $(T(n))$ as the total number of cells that contain all the nodes in a multicast group. Note that \#MEMTC $(T(n))$ also represents the average number of channel uses required to transport a packet from a source to its $m$ destinations in a multicast tree. The following lemma establishes the achievable lower bound for the multicast throughput capacity of MPT and MPR as a function of $\overline{\# \operatorname{MEMTC}(T(n))}$.

Lemma 8: The achievable lower bound of the multicast capacity is given by

$$
C_{m}(n)=\Omega\left(\frac{n T^{2}(n)}{\overline{\# \operatorname{MEMTC}(T(n))}}\right) .
$$

Proof: There are $(T(n) / \sqrt{2})^{-2}$ cells in the unit square network area. From the definition of $\overline{\# \operatorname{MEMTC}(T(n))}$ and the fact that our TDMA scheme does not change the order capacity, it is clear that there are at most in the order of \#MEMTC $(T(n))$ interfering cells for multicast communication. Hence, from Lemma 7, there are a total of $\Theta\left(n^{2} T^{2}(n)\right)$ nodes transmitting simultaneously, which are distributed over all the $(T(n) / \sqrt{2})^{-2}$ cells. Accordingly, the total lower bound capacity is given by $\Omega\left(\left(n^{2} T^{2}(n)\right)(\overline{\text { \#MEMTC }(T(n))})^{-1}\right)$ which is the ratio between the total number of active links at any time divided by the number of channel uses required to complete a multicast communication group. Normalizing this value by total number of nodes in the network, $n$, proves the lemma.

Given the above lemma, to express the lower bound of $C_{m}(n)$ as a function of network parameters, we need to compute the upper bound of $\overline{\# \text { MEMTC }(T(n))}$, which we do next.

Lemma 9: The average number of cells covered by a multicast tree, $\overline{\operatorname{MEMTC}(T(n))}$, is upper bounded as

$$
\overline{\# \operatorname{MEMTC}(T(n))}=O\left(\frac{\sqrt{m}}{T(n)}\right)=O\left(\frac{1}{T(n)}\right) .
$$

Proof: Because $T(n)$ is the transceiver range of the network, the maximum number of cells for this multicast tree must be $O\left(\sqrt{m} T^{-1}(n)\right)$, i.e., $\overline{\# \operatorname{MEMTC}(T(n))}=$ $O\left(\sqrt{m} T^{-1}(n)\right)$. This upper bound can be achieved only if every two adjacent nodes in the multicast tree belong to two different cells in the network. However, in practice, it is possible that some adjacent nodes in multicast tree locate in a single cell. Consequently, this value is upper bound as described in (12). Note that the optimum multicast tree in wireless ad hoc network, may not necessarily cover the same route. However, since our intention is to derive the achievable lower bound, we can design a scheme that follows the MEMT routing, so that each relay in that real routing tree is in the cells which is crossed by MEMT or the neighbor cell of MEMT. Therefore, we count all of those cells which include those real relays as MEMTC (see Fig. 5). Since as we describe later, this technique will provide the same order bound for the capacity as the upper bound, clearly the optimum multicast tree cannot achieve lower order bound.

Combining Lemmas 8 and 9, we arrive at the achievable lower bound of the multicast throughput capacity in dense random wireless ad hoc networks with MPT and MPR.

Theorem 2: When the number of the destinations $m$ is a constant, the achievable lower bound of the multicast throughput capacity with MPT and MPR is

$$
C_{m}(n)=\Omega\left(n T^{3}(n)\right) .
$$

Next, we discuss the routing scheme to achieve the lower bound capacity which is similar to the scheme used in [30]. According to our model, each multicast session creates a multicast tree $\overline{\# \operatorname{MEMT}(T(n))}$ to connect the source and destinations. The trees are denoted as $T_{i} \mathrm{~s}$, where $i=1,2, \cdots, n$. The routing scheme between source and destination is such that packets are forwarded by using cells that are intersected only by $T_{i}$. There is a bound on the number of trees that each cell needs to serve, which means that we can bound the probability that the trees intersects a particular cell. 


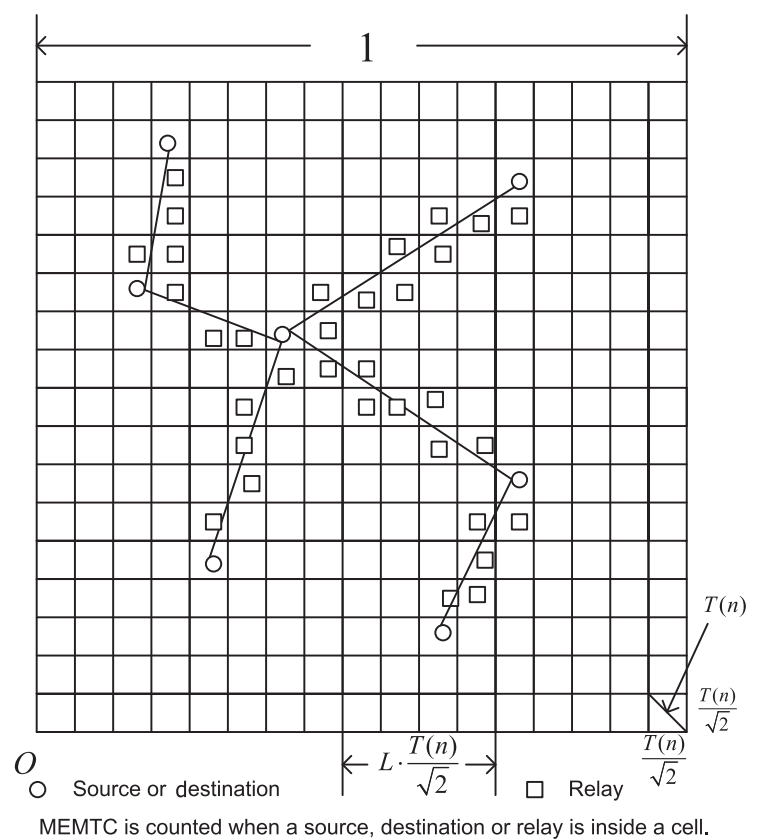

Fig. 5. Illustration of $\overline{\# \operatorname{MEMTC}(T(n))}$, the Euclidean distance of neighbor relay is smaller than $T(n)$.

We will prove the following lemma with MPT and MPR case for multicast communications in the follows.

Lemma 10: We have, for any $T(n)=\Omega(\sqrt{\log n / n})$, $\lim _{n \rightarrow \infty} \operatorname{Prob}\left(\sup _{(k, j)}\left\{\right.\right.$ Number of trees $T_{i}$ s intersecting $\left.S_{k, j}\right\}$ $=O(n T(n)))=1$.

Proof: For every tree $T_{i}$ and cell $S_{k_{0}, j_{0}}$, with Lemma 9,

$$
\begin{aligned}
p & =\operatorname{Prob}\left\{\text { Tree } T_{i} \text { intersects } S_{k_{0}, j_{0}}\right\} \\
& =\Theta\left(T^{2}(n) \overline{\# \operatorname{MEMTC}(T(n))}\right) \\
& =O(T(n)) .
\end{aligned}
$$

First, we bound the number of trees served by one particular cell $S_{k_{0}, j_{0}}$. Define independent identically distributed (i.i.d.) random variables $I_{i}, 1 \leq i \leq n$, as follows:

$$
I_{i}=\left\{\begin{array}{l}
1, \text { if } T_{i} \text { intersects } S_{k_{0}, j_{0}} \\
0, \text { if not. }
\end{array}\right.
$$

Then, $\operatorname{Prob}\left(I_{i}=1\right)=p, \forall i$, where $p$ is defined in (15). Denote by $Z_{n}$ the total number of trees served by $S_{k_{0}, j_{0}}$. Then, $Z_{n}:=I_{1}+I_{2}+\cdots+I_{n}$. Thus, by the Chernoff bounds [25], for all positive $b$ and $a, \operatorname{Prob}\left(Z_{n}>b\right) \leq \frac{E\left[e^{a Z_{n}}\right]}{e^{a b}}$. Because $1+x \leq e^{x}$, we have

$$
\begin{aligned}
E\left[e^{a Z_{n}}\right] & =\left(1+\left(e^{a}-1\right) p\right)^{n} \leq \exp \left(n\left(e^{a}-1\right) p\right) \\
& =O\left(\exp \left(e^{a}-1\right) n T(n)\right)
\end{aligned}
$$

Now, choosing $b=\Theta(n T(n))$, we get $\operatorname{Prob}\left(Z_{n}=\right.$ $\Omega(n T(n)))=O(\exp (n T(n)))$.

Thus by the union bound, we have

Prob (Some cell intersects $\Omega(n T(n))$ trees ) $\leq \sum_{k, j} \operatorname{Prob}\left(\right.$ Cell $S_{j k}$ intersects more than $\Omega(n T(n))$ trees $)$

$=O\left(\frac{1}{T^{2}(n)} \exp (-n T(n))\right)$.

The right hand side tends to zero for $T(n)=\Omega(\sqrt{\log n / n})$ as $n$ goes to infinity.

We know that there exists a transmitting schedule such that in every $L^{2}$ ( $L$ is constant) slots, each cell transmits at rate $W$ bits/second with maximum transmission distance $T(n)$. Therefore, the rate for each cell is $\Theta\left(n^{2} T^{4}(n)\right) W / L^{2}$. From Lemma 10 , each cell needs to transmit at rate $O\left(C_{m}(n) n T(n)\right)$ with probability approaching one. In order to accommodate this requirement by all cells, we need

$$
C_{m}(n) n T(n)=\Omega\left(\left(n^{2} T^{4}(n)\right) W / L^{2}\right) .
$$

Thus, we have proven the achievable throughput for Theorem 2 in order to guarantee each cell can support this capacity. It can be written as

$$
C_{m}(n)=\Omega\left(n T^{3}(n)\right) .
$$

We have proved there is no congestion in relay nodes. Furthermore, we will prove there is not any congestion in destination. Suppose each source selects a destination randomly and in dependently. Then we will prove with high probability, a node can be destination for at most $3 \log n / \log \log n$ sources. This problem is similar to the "bins and balls problems" in [31].

Lemma 11: The probability of a particular destination having $k$ sources selected is

$\lim _{n \rightarrow \infty} \operatorname{Prob}[$ destination $i$ has at least $k$ sources $] \leq\left(\frac{e}{k}\right)^{k}$

Proof: If we look at any subset of sources of size $k$, then the probability that the subset of sources select destination $i$ is $\left(\frac{1}{k}\right)^{k}$. We then take a union bound of these probabilities over all $\left(\begin{array}{l}n \\ k\end{array}\right)$ subsets of size $k$. The events we are summing over, though, are not disjoint. Therefore, we can only show that the probability of a destination having at least $k$ balls is at most $\left(\begin{array}{l}n \\ k\end{array}\right)\left(\begin{array}{c}n \\ 1\end{array}\right)^{k}$. Using Stirling's approximation $\lim _{n \rightarrow \infty} \frac{n !}{\sqrt{2 \pi n}\left(\frac{n}{e}\right)^{n}}=1$, we have

$$
\lim _{n \rightarrow \infty} \frac{\left(\begin{array}{l}
n \\
k
\end{array}\right)\left(\begin{array}{c}
n \\
1
\end{array}\right)^{k}}{\left(\frac{e}{k}\right)^{k}}=1
$$

which proved the lemma.

Lemma 12: With high probability, i.e., with probability greater than $1-1 / n$, there exist at most $3 \log n / \log \log n$ sources for each destination.

Proof: Let $k=3 \log n / \log \log n$. From Lemma 11, we have

$$
\begin{aligned}
& \lim _{n \rightarrow \infty} \operatorname{Prob}[\text { destination } i \text { has at least } k \text { sources] } \\
\leq & \left(\frac{e}{k}\right)^{k}=\left(\frac{e \log \log n}{3 \log n}\right)^{\frac{3 \log n}{\log \log n}} \\
\leq & \exp \left(\frac{3 \log n}{\log \log n}(\log \log \log n-\log \log n)\right) \\
= & \exp \left(-3 \log n+\frac{3 \log n \log \log \log n}{\log \log n}\right)
\end{aligned}
$$




$$
\leq \exp (-2 \log n)=\frac{1}{n^{2}}
$$

Using union bound, we have

$$
\begin{aligned}
& \lim _{n \rightarrow \infty} \operatorname{Prob}[\text { any destination has at least } k \text { sources] } \\
& \leq n \frac{1}{n^{2}}=\frac{1}{n}
\end{aligned}
$$

which implies that

$$
\lim _{n \rightarrow \infty} \operatorname{Prob}[\text { all destinations have at most } k \text { sources }] \geq 1-\frac{1}{n} \text {. }
$$

It proved the lemma.

For MPTR unicast, we require that for all destinations, there does not exist any one whose traffic load congestion is larger than the total throughput it can support. It means that the maximum throughput for each destination should always be greater than the total traffic load. In MPTR case, the total throughput of each destination is $n T^{2}(n)$. The traffic load congestion for each destination is the multiplication of throughput per node of $n T^{3}(n)$ and the maximum possible sources that select a node, i.e., $3 \log n / \log \log n$. Hence,

$$
n T^{2}(n)>n T^{3}(n) \frac{3 \log n}{\log \log n},
$$

which is

$$
T(n)=O\left(\frac{\log \log n}{\log n}\right) .
$$

Therefore, $T(n)$ is bounded as

$$
\Omega\left(\sqrt{\frac{\log n}{n}}\right)=T(n)=O\left(\frac{\log \log n}{\log n}\right) .
$$

The left side is the connectivity constraint and the right side is the traffic load constraint to guarantee that $C(n)=n T^{3}(n)$ can be achieved.

\section{Tight Bound and Comparison with Point-to-Point Communi- cation}

From Theorems 1 and 2, we can provide a tight bound throughput capacity for multicasting when nodes have MPT and MPR capabilities in dense random wireless ad hoc networks as follows.

Theorem 3: The throughput capacity of multicast with constant number $m$ destinations (i.e., $m$ is not a function of $n$ ) in a random dense wireless ad hoc network with MPT and MPR is

$$
C_{m}^{\mathrm{MPT}+\mathrm{MPR}}(n)=\Theta\left(n T^{3}(n)\right) .
$$

The transceiver range of MPT and MPR should satisfy $\Omega\left(\sqrt{\frac{\log n}{n}}\right)=T(n)=O\left(\frac{\log \log n}{\log n}\right)$.

Following similar proof procedure, we can derive the tight capacity for MPT or MPR only in the following theorem, which has been shown in [32].
Theorem 4: The throughput capacity of multicast with constant $m$ number of destinations in a random dense wireless ad hoc network with MPT or MPR is given by

$$
C_{m}^{\mathrm{MPT}}(n)=C_{m}^{\mathrm{MPR}}(n)=\Theta(T(n)) .
$$

The transceiver range of MPT and MPR should satisfy $\Omega\left(\sqrt{\frac{\log n}{n}}\right)=T(n)=O\left(\frac{\log \log n}{\log n}\right)$.

The multicast throughput capacity with point-to-point communication is given by the following lemma [26].

Lemma 13: In multicast with a constant $m$ number of destinations, without MPR or MPR ability, the capacity is

$$
C_{m}^{\mathrm{PTP}}(n)=\Theta\left(\frac{1}{n r(n)}\right)
$$

where $r(n)=\Omega(\sqrt{\log n / n})$ and PTP means point-to-point communication. When $r(n)=\Theta(\sqrt{\log n / n})$ for the minimum transmission range to guarantee the connectivity, then we obtain the maximum capacity as $C_{m}^{\text {PTP-Max }}(n)=\Theta(1 / \sqrt{n \log n})$.

Combining Theorem 3 with Lemma 13, the gain of throughput capacity with MPT and MPR capability in wireless ad hoc networks can be stated as follows.

Theorem 5: In multicast with a constant $m$ number of destinations, with MPT and MPR ability, the gain of pernode throughput capacity compared with point-to-point communication is $\Theta\left(n^{2} T^{4}(n)\right.$ (with only MPT or MPR, the gain is $\Theta\left(n T^{2}(n)\right)$, where $\Omega(\sqrt{\log n / n})=T(n)=r(n)=$ $O(\log \log n / \log n)$. When $T(n)=\Theta \sqrt{\log n / n})$, the gain of per-node capacity is at least $\Theta\left(\log ^{2} n\right)$ (with only MPT or MPR, the gain is $\Theta(\log n))$.

\section{CAPACITY WITH NC, MPT AND MPR WITH FINITE $M$}

We now study the multi-source multicast capacity of a wireless network when nodes use NC, MPT and MPR. The results we present serve as an upper-bound for what can be achieved by combining NC, MPT and MPR in the presence of interference. Our arguments are generic and can be used to deduce upper bounds for the multicast capacity of other interesting cases where NC is used along with only one of MPT or MPR, or even the scenario where $\mathrm{NC}$ is used with traditional point-to-point communication.

In the proof, we use the characteristic of network coding which does not change the capacity information flow across the sparsity cut and has been widely used in [9] and [18].

We deduce the bounds for the case of multi-source multicasting by reducing it to a suitable unicast routing problem. Under the reduction, an upper bound for the unicast problem also serves for the original multicast routing problem. Thus consider the following simple yet powerful lemma.

Lemma 14: Consider a network with $n$ nodes $V=$ $\left\{a_{1}, \ldots, a_{n}\right\}$ and $k$ multicast sessions. Each session consists of one of the $n$ nodes acting as a source with an arbitrary finite subset of $V$ acting as the set of destinations. Let $s_{i}$ be the source of the $i$ th session and let $D_{i}=\left\{d_{i 1}, \ldots, d_{i m_{i}}\right\}$ be the set of $m_{i}$ destinations. Let $\lambda=\left[\lambda_{1}, \ldots \lambda_{k}\right]$ be a feasible rate vector, i.e., 


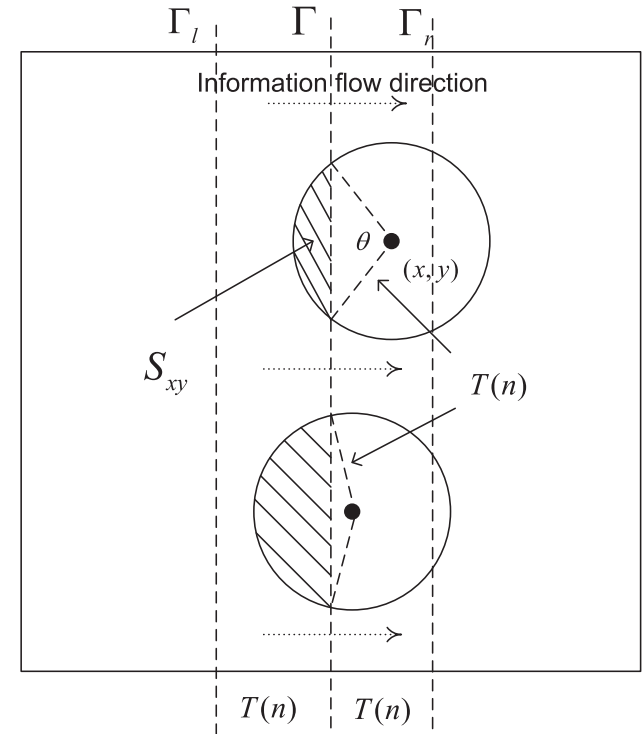

Fig. 6. For a receiver at location $(x, y)$, all the nodes in the shaded region $S_{x y}$ can send a message successfully and simultaneously.

there exists a joint routing-coding-scheduling scheme that can realize a throughput of $\lambda_{i}$ for the $i^{\text {th }}$ session. Then $\lambda$ is also a feasible vector for any unicast routing problem in the same network such that the traffic consists of $k$ unicast sessions with $s_{i}$ being the source of the $i$ th session and the destination $d_{i}$ is any arbitrary element of the set $D_{i}$.

If a multicast capacity from a source to multiple destinations is feasible, then clearly it is feasible to achieve the same capacity to any one arbitrarily chosen node from this set of destinations.

Lemma 15: Consider a random geometric network with $n$ nodes distributed uniformly in a unit square. Consider a decomposition of the unit-square into two disjoint regions $R$ and $R^{c}$ such that the area of each region is of order $\Theta(1)$. Now, consider a multicast traffic scenario consisting of $n$ sessions with each node being the source of a session and $m$ randomly chosen nodes being the destination of the session. We say that a source satisfies property $P$ if the source belongs to region $R$ and at least one of its destination belongs to $R^{c}$ OR if the source belongs to region $R^{c}$ and at least one of its destination belongs to $R$. It can be easily shown that the number of sources satisfying property $P$ are $\Theta(n)$.

\section{Definition 8: Sparsity Cut for Random Networks}

In graph theory, a cut $\Gamma$ is a partition of the vertices (i.e., nodes in the wireless networks) into two sets. The cut capacity is defined to be the sum of bandwidth of the edges crossing the cut. A min-cut is a cut whose capacity is the minimum value of all cuts. In wireless networks, we use sparsity cut instead of mincut [9]. In a unit square area, the cut length $l_{\Gamma}$ is defined as the length of the cut line segment. The cut line segment that we consider have zero width such that no node lies on it. A sparsity cut for a random network is defined as a cut induced by the line segment with the minimum length that separates the region into two subregions. For the square deployment region illustrated in Fig. 6, the middle line induces a sparsity cut.

Since nodes are uniformly deployed in a random network, a sparsity cut captures the traffic bottleneck of these random net- works. The cut capacity represents the information rate that the nodes from one side of the cut as a whole can deliver to the nodes at the other side. This is the maximum information (bits per second) that can be transmitted across the cut from left to right (or from right to left). The sparsity cut capacity is upper bounded by deriving the maximum number of simultaneous transmissions across the cut.

Lemma 16: The capacity of a sparsity cut $\Gamma$ for a unit region has an upper bound of $O\left(T^{-1}(n)\right), O(n T(n))$, and $O\left(n^{2} T^{3}(n)\right)$ for point-to-point communication with network coding, MPT or MPR with network coding and MPT+MPR with network coding respectively.

Proof: The capacity for the point-to-point communication with network coding has been derived in [9] as $O\left(T^{-1}(n)\right)$. According to the protocol model of [1], the disks of radius $T(n)$ centered at each receiver are disjoint. This fact has been utilized in [9]. However, [1] does not consider many-to-one (or one-tomany) communications, which is the case for MPR (or MPT) scheme. Hence, we need some additional arguments to prove the remaining claims.

Let us consider the combination of only MPR with NC. The cut capacity is upper bounded by the maximum number of simultaneous transmissions across the cut. It is easy to see in Fig. 6 that all the nodes located in the shaded area $S_{x y}$ can send their packets to the receiver node located at $(x, y)$. These nodes lie in the left side of the cut $\Gamma$ within an area called $S_{x y}$ and the assumption is that all these nodes are sending packets to the right side of the cut $\Gamma$. For a node at location $(x, y)$, any node in the disk of radius $T(n)$ can transmit information to this receiver simultaneously and the node can successfully decode those packets. In order to obtain an upper bound, we only need to consider edges that cross the cut. Let's first consider all possible nodes that can transmit to the receiver node in the $S_{x y}$ region. The average number of transmitters located in $S_{x y}$ is $n S_{x y}$. The number of nodes that are able to transmit at the same time from left to right is upper bounded as a function of $S_{x y}$.

The area of $S_{x y}$ is computed as

$$
\begin{aligned}
S_{x y} & =\frac{\theta}{2 \pi} \pi T^{2}(n)-T^{2}(n) \sin \left(\frac{\theta}{2}\right) \cos \left(\frac{\theta}{2}\right) \\
& =\frac{1}{2} T^{2}(n)(\theta-\sin \theta) .
\end{aligned}
$$

This area is maximized when $\theta=\pi$.

$$
\max _{0 \leq \theta \leq \pi}\left[S_{x y}\right]=\frac{1}{2} \pi T^{2}(n) .
$$

For the case of MPR (or MPT) with network coding, the disk with radius $T(n)$ centered at any receiver(transmitter) should be disjoint from the other disks centered at the other receivers [9], [18]. Thus, the total number of nodes that can send packets across the cut is upper bounded as

$$
\frac{l_{\Gamma}}{(2+\Delta) T(n)} \frac{1}{2} \pi T^{2}(n) n=c_{1} l_{\Gamma} n T(n)
$$

where $c_{1}=\pi / 2(2+\Delta)$. Similar arguments can be used to bound the cut-capacity when only MPT is combined with NC. 
Now, let us consider the case of combining MPT+MPR with network coding. We utilize the fact that each node has a maximum of $\Theta\left(n T^{2}(n)\right)$ neighbors, which implies that each node can simultaneously receive packets from a maximum of $\Theta\left(n T^{2}(n)\right)$ transmitters. Moreover, any node that receives transmission from across the cut $\Gamma$ has to lie in the region enclosed by the dotted line $\Gamma_{r}$ on the right side in Fig. 6, where $\Gamma_{r}$ is at a $T(n)$ from $\Gamma$. Thus, the rectangular region enclosed by $\Gamma$ and $\Gamma_{r}$ has an area of $\Theta(T(n))$. Hence, an average of $\Theta(n T(n))$ nodes can receive packets from across the cut. Therefore, the total number of transmissions across the cut are bounded by

$$
O\left(n T(n) n T^{2}(n)\right)=O\left(n^{2} T^{3}(n)\right)
$$

Theorem 6: In a wireless ad hoc network formed by $n$ nodes distributed randomly in a unit square with traffic formed by each node acting as source for a multicast sessions with $m=\Theta(1)$ randomly chosen nodes as destinations, the per-session multicast capacities are

$$
\begin{aligned}
& C_{m}^{\mathrm{NC}+\mathrm{PTP}}=O\left(\frac{1}{n T(n)}\right), \\
& C_{m}^{\mathrm{NC}+\mathrm{MPT}}=C_{m}^{\mathrm{NC}+\mathrm{MPR}}=O(T(n)), \\
& C_{m}^{\mathrm{NC}+\mathrm{MPT}+\mathrm{MPR}}=O\left(n T^{3}(n)\right)
\end{aligned}
$$

where NC + PTP denotes the use of NC with point-to-point communication (no MPT or MPR), i.e., a node can only transmit or receive at most one packet at a time.

Proof: For any sparsity cut of the unit area, Lemmas 15 and 14 tell us that we can construct a unicast routing problem satisfying the property that any rate for the unicast problem is feasible for the original multicast problem and we have $\Theta(n)$ source-destination pairs across the cut. Thus, the capacity of the sparsity cut provides a bound for the unicast problem, which can in turn be used to provide an upper bound for the multicast problem. From Lemma 16, finally, we can extend such arguments to show that the combination of NC+MPT (or NC+MPR) and $\mathrm{NC}+\mathrm{MPT}+\mathrm{MPR}$ allows us to simultaneously transmit a maximum of $O(n T(n))$ and $O\left(n^{2} T^{3}(n)\right)$ packets across the cut respectively. The result of the theorem then follows from the fact that the cut capacity has to be divided among the $\Theta(n)$ source-destination pairs across the cut. Finally, because multicast capacity must be upper bounded by the unicast capacity which finishes the proof.

\section{CONCLUSION}

By combining the results from Theorems 3 and 6, the main contribution of this paper is stated in the following theorem.

Theorem 7: In wireless ad hoc networks with multi-pair multicast sessions and with a finite number of $m$ destinations for each source, the throughput capacity utilizing NC, MPT and MPR capabilities for all nodes is the same order as when the nodes are endowed only with MPT and/or MPR

$$
\begin{aligned}
C_{m}^{\mathrm{MPT}+\mathrm{NC}}(n) & =C_{m}^{\mathrm{MPT}}(n), \\
C_{m}^{\mathrm{MPR}+\mathrm{NC}}(n) & =C_{m}^{\mathrm{MPR}}(n),
\end{aligned}
$$

$$
C_{m}^{\mathrm{MPT}+\mathrm{MPR}+\mathrm{NC}}(n)=C_{m}^{\mathrm{MPT}+\mathrm{MPR}}(n) .
$$

Proof: Because from Theorems 3 and 1, we realize the multicast capacity of NC with MPT and MPR is tightly bounded by the tight bound of multicast capacity of MPT and MPR without NC, so the multicast capacity of MPT and MPR with or without NC are the same.

It is also important to emphasize that, as Theorem 6 shows, $\mathrm{NC}$ does not provide any order capacity gain for multi-source multicasting when the size of receiver groups is $m=\Theta(1)$ and nodes use point-to-point communication. Hence, the result in Theorem 7 implies that NC does not provide an order capacity gain when MPT or MPR or combination of MPR and MPT is used, and that MPT and MPR are the real contributing factors for order capacity increases in wireless ad hoc networks.

Finally, it is not clear if there is any gain in multicast communications when only NC is used in point-to-point communication scenario and the number of destinations in a multicast group, $m$, is a function of $n$. This problem is important and will be the subject of future investigation in identifying the actual order capacity contribution of NC in wireless ad hoc networks. It is also important to note that NC provides many other advantages in random wireless ad hoc networks for different applications such as secrecy that are not investigated in this paper.

\section{REFERENCES}

[1] P. Gupta and P. R. Kumar, "The capacity of wireless networks," IEEE Trans. Inf. Theory, vol. 46, no. 2, pp. 388-404, 2000.

[2] M. Franceschetti, O. Dousse, D. Tse, and P. Thiran, "Closing the gap in the capacity of wireless networks via percolation theory," IEEE Transa. Inf. Theory, vol. 53, no. 3, pp. 1009-1018, 2007.

[3] R. M. de Moraes, H. R. Sadjadpour, and J. J. Garcia-Luna-Aceves, "Manyto-many communication: A new approach for collaboration in manets," in Proc. IEEE INFOCOM, Anchorage, Alaska, USA., May 6-12 2007.

[4] A. Ozgur, O. Leveque, and D. Tse, "Hierarchical cooperation achieves optimal capacity scaling in ad hoc networks," IEEE Trans. Inf. Theory, vol. 53, no. 10, pp. 2549-3572, 2007.

[5] R. Ahlswede, C. Ning, S.-Y. R. Li, and R. W. Yeung, "Network information flow," IEEE Trans. Inf. Theory, vol. 46, no. 4, pp. 1204-1216, 2000.

[6] S.-Y. R. Li, R. W. Yeung, and N. Cai, "Linear network coding," IEEE Trans. Inf. Theory, vol. 49, no. 2, pp. 371-381, 2003.

[7] R. Koetter and M. Medard, "An algebraic approach to network coding," IEEE/ACM Trans. Netw., vol. 11, no. 5, pp. 782-795, 2003.

[8] S. H. Lim, Y.-H. Kim, A. E. Gamal, and S.-Y. Chung, "Noisy network coding," submitted to IEEE Trans. Inf. Theory, 2010.

[9] J. Liu, D. Goeckel, and D. Towsley, "Bounds on the gain of network coding and broadcasting in wireless networks," in Proc. IEEE INFOCOM, Anchorage, Alaska, USA., May 6-12 2007.

[10] S. Katti, S. Gollakota, and D. Katabi, "Embracing wireless interference: Analog network coding," in Proc. ACM SIGCOMM, Kyoto, Japan, Aug. 27-31 2007.

[11] S. Zhang, S. Liew, and P. P. Lam, "Hot topic: Physical-layer network coding," in Proc. ACM MobiCom, Los Angeles, California, USA., Sept. 23-29 2006.

[12] A. Ramamoorthy, J. Shi, and R. Wesel, "On the capacity of network coding for random networks," IEEE Trans. Inf. Theory, vol. 51, no. 8, pp. 28782885, 2005.

[13] S. A. Aly, V. Kapoor, J. Meng, and A. Klappenecker, "Bounds on the network coding capacity for wireless random networks," in Proc. NetCod, San Diego, California, USA, Jan. 292007.

[14] Z. Kong, S. A. Aly, E. Soljanin, E. M. Yeh, and A. Klappenecker, "Network coding capacity of random wireless networks under a signal-tointerference-and noise model," Submitted to IEEE Trans. Inf. Theory, 2007.

[15] C. Peraki and S. Servetto, "On the maximum stable throughput problem in random networks with directional antennas," in Proc. ACM MobiHoc, Annapolis, Maryland, USA., June 1-3 2003.

[16] S. Yi, Y. Pei, and S. Kalyanaraman, "On the capacity improvement of ad 
hoc wireless networks using directional antennas," in Proc. ACM MobiHoc, Annapolis, Maryland, USA., June 1-3 2003.

[17] S. Toumpis and A. J. Goldsmith, "Capacity regions for wireless ad hoc networks," IEEE Trans. Wireless Commun., vol. 2, no. 4, pp. 736-748, 2003.

[18] J. J. Garcia-Luna-Aceves, H. R. Sadjadpour, and Z. Wang, "Challenges: Towards truly scalable ad hoc networks," in Proc. ACM MobiCom, Montreal, Quebec, Canada, Sept. 9-14 2007.

[19] Z. Li and B. Li, "Network coding in undirected networks," in Proc. CISS, Princeton, NJ, USA., Mar. 17-19 2004.

[20] Z. Li, B. Li, and L. Lau, "On achieving maximum multicast throughput in undirected networks," IEEE/ACM Trans. Special Issue Netw. Inf. Theory, vol. 52, pp. 2467-2485, 2006.

[21] Z. Wang, S. Karande, H. Sadjadpour, and J. J. Garcia-Luna-Aceves, "On the capacity improvement of multicast traffic with network coding," in Proc. IEEE MILCOM, San Diego, California, USA, Nov. 17-19 2008.

[22] S. Karande, Z. Wang, H. Sadjadpour, and J. J. Garcia-Luna-Aceves, "Multicast throughput order of network coding in wireless ad-hoc networks," in Proc. IEEE SECON, Rome, Italy, June 22-26 2009.

[23] _ "Network coding does not change the multicast throughput order of wireless ad hoc networks," in Proc. IEEE ICC, Dresden, Germany, June 14-18 2009.

[24] M. Steele, "Growth rates of euclidean minimal spanning trees with power weighted edges," The Annals of Probability, vol. 16, no. 4, pp. 1767-1787, 1988.

[25] R. Motwani and P. Raghavan, Randomized Algorithms. Cambridge University Press, 1995.

[26] Z. Wang, H. R. Sadjadpour, and J. J. Garcia-Luna-Aceves, "A unifying perspective on the capacity of wireless ad hoc networks," in Proc. IEEE INFOCOM 2008, Phoenix, Arizona, USA., Apr. 13-18 2008.

[27] X.-Y. Li, S.-J. Tang, and O. Frieder, "Multicast capacity for large scale wireless ad hoc networks," in Proc. ACM MobiCom, Montreal, Canada, Sept. 9-14 2007.

[28] A. Giridhar and P. R. Kumar, "Computing and communicating functions over sensor networks," IEEE J. Sel. Areas Commun., vol. 23, no. 4, pp. 755-764, 2005.

[29] S. Kulkarni and P. Viswanath, "A deterministic approach to throughput scaling wireless networks," IEEE Trans. Inf. Theory, vol. 50, no. 6, pp. 1041-1049, 2004

[30] F. Xue and P. Kumar, Scaling Laws for Ad-Hoc Wireless Networks: An Information Theoretic Approach. Now Publishers Inc., 2006.

[31] J. Kleinberg and E. Tardos, Algorithm Design. Addison Wesley, 2005.

[32] Z. Wang, H. R. Sadjadpour, and J. J. Garcia-Luna-Aceves, "Capacitydelay tradeoff for information dissemination modalities in wireless networks," in Proc. IEEE ISIT, Toronto, Ontario, Canada, July 6-11 2008.

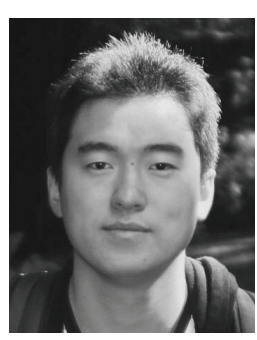

Zheng Wang received the B.S. and M.S. degrees in Electrical Engineering from Peking University, China in 2003 and 2006, respectively and the Ph.D. degree in Electrical Engineering from University of California, Santa Cruz in 2010. He is the recipient of the IEEE Fred W. Ellersick Award for Best Unclassified Paper at MILCOM 2008 and the Best Paper Award at the European Wireless 2010.

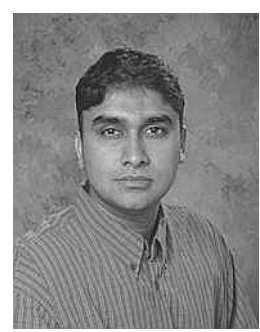

Shirish S. Karande received his B.E. degree in Electronic and Telecommunications Engineering from the University of Pune, India, in 2000 and his M.S. and Ph.D degrees in Electrical Engineering from the Michigan State University in 2003 and 2007, respectively. He is currently a Scientist with the Tata Research Development and Design Centre, India and has previously worked with Philips Research and University of California-Sant Cruz. His research interests include information theory, algorithms and optimization, and machine learning and their applications to network and visual sciences. He is the co-recipient of IEEE Fred W. Ellersick 2008 MILCOM Award for best unclassified paper. While at Michigan State University, he was the Recipient of the Most Outstanding Graduate Student of Electrical and Computer Engineering Award, for the year 2007, and the Excellence in Teaching Award, for year 2005.

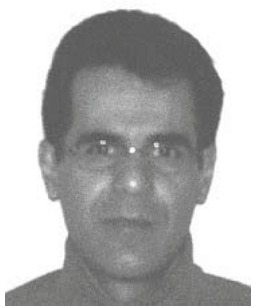

Hamid R. Sadjadpour received his B.S. and M.S. degrees from Sharif University of Technology with high honor and Ph.D. degree from University of Southern California in 1986, 1988, and 1996, respectively. After graduation, he joined AT\&T as a Member of Technical Staff, later Senior Technical Staff Member, and finally Principal Member of Technical Staff in Florham Park, NJ until 2001. In fall 2001, he joined University of California, Santa Cruz (UCSC) where he is now a Professor. He has served as Technical Program Committee Member in numerous conferences and as Chair of Communication Theory Symposium at WirelessCom 2005, and Chair of Communication and Information Theory Symposium at IWCMC 2006, 2007, and 2008 conferences. He has been also Guest Editor of EURASIP on Special Issue on Multicarrier Communications and Signal Processing in 2003 and special issue on Mobile Ad Hoc Networks in 2006, and is currently Associate Editor for Journal of Communications and Networks (JCN). He has published more than 140 publications. His research interests include scaling laws for wireless ad hoc networks, performance analysis of ad hoc and sensor networks, design of MAC and routing protocols for MANETs, and study of interaction between social and wireless communication networks. He is the co-recipient of International Symposium on Performance Evaluation of Computer and Telecommunication Systems (SPECTS) 2007 Best Paper Award, the IEEE Fred W. Ellersick Award for Best Unclassified Paper at the 2008 Military Communications (MILCOM) conference, and Best Paper Award at the European Wireless 2010 conference. He holds more than 13 patents, one of them accepted in spectrum management of T1.E1.4 standard.

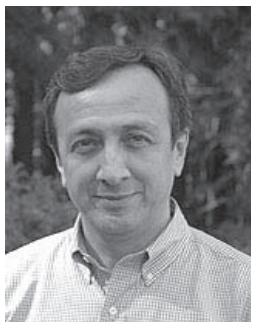

J.J. Garcia-Luna-Aceves received the B.S. degree in Electrical Engineering from the Universidad Iberoamericana, Mexico City, Mexico in 1977; and the M.S. and Ph.D. degrees in Electrical Engineering from the University of Hawaii at Manoa, Honolulu, HI in 1980 and 1983, respectively. He holds the Jack Baskin Endowed Chair of Computer Engineering at the University of California, Santa Cruz (UCSC), is Chair of the Computer Engineering Department, and is a Principal Scientist at the Palo Alto Research Center (PARC). Prior to joining UCSC in 1993, he was a Center Director at SRI International (SRI) in Menlo Park, California. He has been a Visiting Professor at Sun Laboratories and a Principal of Protocol Design at Nokia. He holds 35 U.S. patents, and has published three books and more than 400 journal and conference papers. He has directed $30 \mathrm{Ph} . \mathrm{D}$. theses and 28 M.S. theses since he joined UCSC in 1993. He has been the General Chair of the ACM MobiCom 2008 Conference; the General Chair of the IEEE SECON 2005 Conference; Program Co-Chair of ACM MobiHoc 2002 and ACM MobiCom 2000; Chair of the ACM SIG Multimedia; General Chair of ACM Multimedia 2003 and ACM SIGCOMM 2008; and Program Chair of IEEE MULTIMEDIA 2002, ACM SIGCOMM 2007, and ACM SIGCOMM 2006. He has served in the IEEE Internet Technology Award Committee, the IEEE Richard W. Hamming Medal Committee, and the National Research Council Panel on Digitization and Communications Science of the Army Research Laboratory Technical Assessment Board. He is an IEEE Fellow and an ACM Fellow, and is listed in Marquis Who's Who in America and Who's Who in The World. He is the co-recipient of the IEEE Fred W. Ellersick 2008 MILCOM Award for best unclassified paper. He is also co-recipient of Best Paper Awards at the European Wireless Conference 2010, IEEE MASS 2008, SPECTS 2007, IFIP Networking 2007, and IEEE MASS 2005 conferences, and of the Best Student Paper Award of the 1998 IEEE International Conference on Systems, Man, and Cybernetics. He received the SRI International Exceptional-Achievement Award in 1985 and 1989. 